\title{
CON FIGURA E VITA PROPRIA. MARÍA ZAMBRANO E LA QUESTIONE DELLA CITTADINANZA DELLE DONNE
}

\author{
ELENA LAURENZI
}

\author{
Universitat de Barcelona; Università del Salento
}

Il saggio esamina gli scritti politici della filosofa María Zambrano insieme ad alcuni testi dedicati alla posizione delle donne nella società e nella cultura, con l'obiettivo di verificarne l'attualità e la rilevanza per il dibattito sulla cittadinanza delle donne che attraversa il femminismo dalla seconda metà del secolo Xx. Con questo obiettivo si mettono in dialogo le pagine di Zambrano con alcuni testi di teoriche contemporanee quali Carol Pateman, M. Luisa Boccia, Carla Lonzi, Marisa Forcina, Françoise Collin, Lia Cigarini, Joan Scott, Marilyn Friedman, attorno a tre nodi tematici: le ambivalenze e i paradossi del concetto di individuo; la sfida dell'amicizia alla divisione tra pubblico e privato; il rapporto tra cittadinanza e lavoro.

PAROle CHIAVE: María Zambrano, cittadinanza, teoria politica feminista, liberalismo, individuo/individualità, amicizia.

\section{Con figura y vida propia. María Zambrano y la cuestión de la ciudadanía de las mujeres}

El ensayo examina los escritos políticos de la filósofa María Zambrano junto con algunos textos dedicados a la posición de la mujer en la sociedad y la cultura, con el objetivo de averiguar su actualidad y relevancia con respecto al debate sobre la ciudadanía de las mujeres que atraviesa el feminismo desde la segunda mitad del siglo XX. Con este objetivo se hacen dialogar las páginas de Zambrano con los textos de algunas teóricas feministas como Carol Pateman, M. Luisa Boccia, Carla Lonzi, Marisa Forcina, Françoise Collin, Lia Cigarini, Joan Scott, Marilyn Friedman, alrededor de tres nudos temáticos: las ambivalencias y paradojas del concepto de individuo; el desafío de la amistad frente a la separación público-privado; la relación entre ciudadanía y trabajo.

PALABRAS ClAVE: María Zambrano, ciudadanía, teoría politíca feminista, liberalismo, individuo/individualidad, amistad.

\section{A life and a figure of its own. María Zambrano and female citizenship}

This essay explores the political writings by the philosopher María Zambrano, as well as some of her writings focused on the place occupied by women in society and culture. The aim is to determine her significance and relevance in today's current debates on women's citizenship, debates which have occupied feminism during the second half of the twentieth century. Using texts by feminist theorists such as Carol Pateman, M. Luisa Boccia, Carla Lonzi, Marisa Forcina, Françoise Collin, Lia Cigarini, Joan Scott, and Marilyn Friedman, the essay examines Zambrano's writings concerning three themes: the ambivalence and paradox of the concept of the 
individual, the challenge of friendship against the separation of public and private spheres, and the relationship between citizenship and work.

KEY WORDS: María Zambrano, citizenship, feminist political theory, liberalism, individual/ individuality, friendship.

\section{Una differenza conquistata}

Tra le grandi filosofe della prima metà del '900, María Zambrano è forse quella che con maggiore lucidità affronta e mette in questione la "scelta del neutro", vale a dire la tacita obbligazione con cui le donne pagarono l'ammissione nel santuario della filosofia al prezzo di ignorare il fatto di essere, appunto, donne, come se questo non avesse alcun rilievo per il pensare. ${ }^{1}$ Anche se Zambrano non si sottrae totalmente a tale ambivalenza, tra le righe delle sue opere più note e soprattutto in un buon numero di saggi poco studiati si può cogliere un'esplicita considerazione della differenza sessuale come questione filosofica e politica. La sua riflessione è radicata nell'esperienza: come "donna filosofo" percepisce la propria anomalia, ed è su questa base che denuncia il carattere "viril" della cultura egemonica e la conseguente "mancanza di sede" delle donne al suo interno (cfr. Zambrano, 1995: 94). Scrivere testi filosofici, significa dunque, per lei, anche conquistare "una sede": un posto, un diritto, un riconoscimento; una forma di cittadinanza. Dall'esperienza - accolta ed elaborata - della differenza sessuale scaturisce, a mio avviso, la potenza dirompente del suo pensiero che sembra penetrare nelle profondità inconfessate della tradizione filosofica per obbligarla a confrontarsi con un'alterità radicale. Se viene riconosciuta pregnanza filosofica ai testi esplicitamente dedicati a quella che allora si chiamava la "questione femminile" (ma che lei non chiama mai così), se ne vengono analizzati gli sviluppi e gli intrecci nel contesto globale dell'opera, si può notare come tutta la riflessione di María Zambrano - nei suoi aspetti ontologici, antropologici, politici, etici, religiosi- attinga profondamente alla consapevolezza della differenza sessuale, e come la filosofa si avvalga di argomenti, concetti e modalità della conoscenza che traggono origine dallesperienza femminile, sia quando intraprende la

\footnotetext{
${ }^{1}$ Di "scelta del neutro"parla Françoise Collin, nel suo contributo alla Storia delle donne curata da G. Duby e M. Perrot, osservando che: "prima del femminismo le poche donne filosofo non hanno affrontato il problema dei sessi, né Jeanne Hersch, né Suzanne Langer o Gisèle Brelet, Jeanne Delhomme, Simone Weil o Edith Stein e neppure Hannah Arendt" (Collin, 1997). L'affermazione di Collin andrebbe comunque sfumata. Tra le pensatrici citate, almeno una, Edith Stein, dedica alla differenza tra i sessi numerose e notevoli pagine. Cfr. Stein, 1987. Si veda a questo proposito anche lo studio di Angela Ales Bello, 1992. Le coincidenze tra le posizioni di Stein e quelle di Zambrano meriterebbero di essere ulteriormente indagate. Un primo approccio alla questione è stato tentato in un seminario tenuto da me e da Angela Lorena Fuster nell'ambito del XXI Congresso Annuale della AILCFH: "Habitar el género Inhabiting Gender", Barcelona 19, 20 e 21 ottobre 2011.
} 
rivisitazione critica della civiltà occidentale e delle categorie della tradizione filosofica, sia quando definisce i lineamenti della propria proposta.

Anche a motivo di questa attenzione alla differenza sessuale, lopera di Zambrano mi sembra specialmente feconda di stimoli e di strumenti per il pensiero filosofico e politico dell'attualità, ed in particolare per il pensiero politico del femminismo, allorché questo pone la questione della cittadinanza femminile come fulcro della sua analisi critica del modello liberale egemonico. La presenza politica delle donne è infatti una sorgente di continui paradossi, che non hanno trovato scioglimento nella acquisizione - almeno nelle democrazie e negli stati di diritto dell'Occidente-dell'uguaglianza formale tra i sessi. Le cifre stanno a dimostrare che, sul piano sostanziale, lo scarto tra uomini e donne (in termini di potere, di presenza pubblica e di disponibilità delle risorse economiche) resta profondo, e la constatazione vale, sia pur con importanti distinguo, per il complesso delle democrazie occidentali. ${ }^{2}$ Dunque proprio la realizzazione formale dell'uguaglianza ne palesa le aporie, come ben avverte Maria Luisa Boccia (2002), parlando del "paradosso della cittadinanza compiuta". Lanalisi di questo paradosso induce a interrogarsi sui limiti costitutivi dei diritti e dello stesso stato di diritto rispetto all'esigenza di libertà espressa dalle donne. Marisa Forcina (2009) scrive a questo proposito che si tratta di una questione "di rappresentazione, più che di rappresentanza", la cui soluzione esige pertanto un lavoro sul piano del simbolico: una discussione delle categorie egemoniche del pensiero a partire dal modello corrente della polis, dei suoi elementi costitutivi e dei suoi presupposti antropologici.

L'obiettivo fondamentale di questo mio intervento è mettere in luce nell'opera di Zambrano l'esistenza di elementi pertinenti e rilevanti per la riflessione politica del femminismo contemporaneo. Presenterò qui solo una sintetica trattazione, in assoluto non esaustiva, di tre di questi elementi, legati dal filo rosso della discussione del concetto di individuo.

\section{Dall'individuo alla persona}

Nel concetto di individuo, perno della teoria politica moderna, le teoriche del femminismo hanno individuato la radice dei paradossi in cui si dibatte la richiesta di cittadinanza da parte delle donne. A cominciare dall'azione pioniera di Olympe de Gouges, questa richiesta si è vista infatti costretta a fare appello a un "universalismo escludente" (Scott, 1996), e a rivendicare la soggettività politica sulla base di un paradigma di cittadino/individuo riassunto nella nota definizione rousseauniana, che lo suppone maschio, proprietario e padre di famiglia. Rispetto

\footnotetext{
${ }^{2}$ La letteratura sulla cittadinanza femminile è molto vasta. Tra i titoli che mi sono serviti di riferimento: Dietz, 1985; Libreria delle donne di Milano, 1987; Bonacchi, Groppi, 1993; Young, 1994; Castells, 1996; Del Re, Heinen, 1996; Bimbi, Del Re, 1997; Birulés, 2014; Forcina, 2009; Forcina, 2003.
} 
a quel modello, che costituisce "il grado zero degli enunciati della cittadinanza, il loro comune referente antropologico" (Boccia, 2002), la differenza femminile è concepita solo come divergenza, deviazione e mancanza. Essa appare priva di significato per il mondo comune, ed assume rilievo solo nella discussione dei "bisogni" specifici delle donne, in rapporto alla cosiddetta "condizione femminile".

In varie occasioni, Zambrano denuncia apertamente la prevaricazione implicita nell'uso del maschile come universale; dietro la neutralità di tutte le categorie che definiscono l'umano coglie la surrettizia auto-rappresentazione della virilità, che "sobre todo en ciertas épocas y de la manera más noble no se ha definido como poder sexual sino como justicia y razón, es decir como objetividad" (Zambrano, 1940b: 367). Questa consapevolezza va messa a mio avviso in relazione con la critica all'individualismo che Zambrano avanza nel suo primo libro, Horizontes del Liberalismo, scritto nel 1928. Mettendo a fuoco la nozione d'individuo, la giovane autrice ne denuncia il carattere astratto e sterile, paragonandolo a quello delle categorie pure del pensiero kantiano: nell'individuo, scrive, l'essere umano, avulso dalla condizione corporea, incarnata, si riduce a un "arquetipo" in cui "ningún hombre carnal vivo podría reconocerse" (Zambrano, 1996: 241). Essa è pertanto inadeguata a dar conto e a farsi carico della condizione umana nella sua pluralità, materialità e contingenza, che porta inscritta in sé la differenza sessuale.

La critica alla deriva individualistica del liberalismo politico ed economico coesiste tuttavia in quest'opera con un sostanziale apprezzamento della filosofia liberale, che Zambrano difende contro gli attacchi degli ideologi del fascismo, quale baluardo della dignità umana, proprio in ragione del suo "amor por el individuo". Anche tale difesa porta, a mio avviso, i segni di una coscienza femminile. Come donna, infatti, non le sfuggiva la portata rivoluzionaria dell'affermazione dell'essere umano singolare e unico come valore in sé, al di sopra di ogni vincolo di "tribu", "casta", o "sangre" (Zambrano, 2006). Ed è appunto il riconoscimento dell'individualità che negli anni quaranta — nei saggi "Eloisa o la existencia de la mujer" (1945), "La mujer en la historia" (1943), "A propósito de la "grandeza y servidumbre de la mujer»" (1947) o nelle conferenze dedicate a "La mujer y la cultura" (1940) rivendica per sé e per tutte le donne. Si tratta tuttavia di una rivendicazione che non coincide con l'assimilazione al modello antropologico maschile, ma con la possibilità di venir riconosciute nella singolarità unica ed irripetibile di esseri umani: di emergere dal "mundo de lo sagrado" per esistere "a la manera masculina, con figura y vida propia" (Zambrano, 1995:100). La soggettività morale e politica delle donne si esprime dunque nei termini di una sovranità intesa non come dominio e controllo sulla realtà, bensì come autodeterminazione: sovranità sulla propria esistenza e sulla sua rappresentazione. A questa duplice dimensione mi pare che rimandino i due termini usati da Zambrano: "vida" e "figura". 
La tensione semantica che si percepisce, in questi scritti, tra i concetti di "individuo" e "individualità" riflette, a mio parere, la consapevolezza da parte di Zambrano dell'ambivalenza del termine "individuo" e delle conseguenze complesse del suo sostanziarsi al maschile. Da un lato, infatti, "individuo" è il prototipo antropologico su cui si fonda la cittadinanza di cui si è detto, e le donne sono state per molto tempo escluse dal godimento dei diritti "naturali" con l'argomento che non corrispondevano ai suoi requisiti. Ma, a complicare la faccenda, "individuo" va anche inteso nell'accezione di essere umano unico e distinto: persona singolare, intera (in-divisa) ed originale. Zambrano è pienamente consapevole del fatto che le donne, definite come gruppo sulla base di una differenza derivata e subordinata rispetto al prototipo, mancano del riconoscimento dello status di individuo anche in questa seconda accezione. Sul piano politico come sul piano morale e culturale, esse non sono considerate come "individue" o "soggette" (cfr. Forcina, 2000), ma vengono contemplate nei termini generici, secondo i quali ognuna di esse riproduce i caratteri della specie. ${ }^{3} \mathrm{E}$ questa la ragione per cui la battaglia delle donne per la cittadinanza è stata, in primo luogo, una battaglia per il riconoscimento della propria individualità, di una autonomia morale, ancor prima che giuridica: "In gioco, nelle lotte per la cittadinanza, non è mai stata solo l'appartenenza alla polis, ma quella all'umanità" (Boccia, 2002: 25).

La riflessione sull'individualità approda, in Persona $y$ democracia, alla categoria di "persona", che Zambrano rielabora con differenze rilevanti rispetto al personalismo. Nella sua trattazione possiamo cogliere la proposta di un modello di soggettività politica connotata dai caratteri dell'originalità e dell'unicità, alternativa all'individualismo astratto del contrattualismo, ma distante anche dalla concezione identitaria dell'agente politico connotato dall'appartenenza a un collettivo (classe, comunità etnica o nazionale, genere, e il lungo e trito "eccetera"). La linea scelta da Zambrano richiama fortemente il pensiero di Hannah Arendt. La persona è infatti concepita come una singolarità unica e irripetibile, un "qualcuno" che trascende i contenuti sociali e identitari che definiscono ciò che è socialmente senza tuttavia prescindere da essi: autore, e non semplice attore, della vita (cfr. Arendt, 1999; Id. 2009). La trascendenza del particolare nella dimensione politica non coincide con la presunta quanto impossibile astrazione dalle determinazioni storico-sociali che ogni essere umano porta in sé, ma consiste nella rielaborazione originale di questo "dato" (di questo "dono ricevuto", come lo pensa Arendt) che produce qualcosa di nuovo. ${ }^{4} \mathrm{La}$ soggettività politica si contraddistingue dunque per il suo carattere "augurale" e insieme concreto, incarnato. Per Zambrano come per Arendt, infatti, la politica radica nella condizione della natalità. Persona è il nuovo che ogni essere umano

\footnotetext{
${ }^{3}$ Per una discussione di questi temi cfr. Collin, 1992.

${ }^{4}$ Cfr. Collin,1992b ; Birulés, 2007; Id., 2012.
} 
rappresenta venendo al mondo, l'inedito che appare. E', dunque, un'identità non oggettivabile, anche perché è intrinsecamente relazionale. Si manifesta e si rivela solo nella convivenza, nella forma della narrazione di sé — un ordito del proprio essere e della propria storia, che Zambrano concepisce nei termini agostiniani della confessione- e di un agire che è, anch'esso, esporsi agli altri (dar la cara), e assumere la responsabilità del proprio stare al mondo.

Nel pensiero politico di María Zambrano, la categoria di persona è alla base di una rielaborazione innovativa dell'idea di democrazia, a partire dal presupposto del pluralismo che ne è un connotato fondamentale. In Persona y democracia è possibile vedere come Zambrano operi uno spiazzamento radicale della discussione politica: dalla teoria della governabilità e del diritto, questa passa all'analisi delle condizioni e delle forme della convivenza e della comunicazione. La sua riflessione approda a una concezione musicale della democrazia contrapposta alla fissità strutturale della concezione architettonica predominante: "Y la sinfonía hemos de escucharla, actualizarla cada vez; hemos de rehacerla en cierto modo, o sostener su hacerse: es una unidad, un orden que se hace ante nosotros y en nosotros. Nos exige participación. Hemos de entrar en él para recibirlo" (Zambrano, 2006: 206). Si riassume in questo passo una concezione non formale del regime democratico: la piena adesione all'ideale di democrazia partecipativa che anche oggi ci appare come la sola possibile soluzione alla grave crisi attraversata dalle forme tradizionali della politica. L'immagine della sinfonia trova un'interessante rispondenza nelle pagine di Marisa Forcina (2003), che ricorre alla stessa metafora musicale per riassumere i caratteri di un regime politico capace di accogliere e comporre le differenze concrete e incarnate degli esseri umani. La politica viene assimilata a un concerto in cui confluiscono una pluralità di voci che sgorgano da spazi eterogenei, e che non possono essere ricondotte a una grammatica omologante, come nelletica della comunicazione di stampo habermasiano, né fissate in recinti ermeneutici impenetrabili, come in alcune teorie del multiculturalismo politico. ${ }^{5}$

\section{Per una politica dell'amicizia}

Nelle loro elaborazioni politiche, le donne hanno eluso i sistemi contrapposti del contrattualismo e del comunitarismo, denunciandoli entrambi incapaci, per motivi speculari, di dar conto della differenza incarnata nella molteplicità delle esistenze. ${ }^{6}$ Lattenzione si è spostata invece sulle "comunità di elezione" (Friedman, 1993), in quanto luoghi ove si sperimentano modalità di convivenza e

\footnotetext{
${ }^{5}$ Forcina fa riferimento anche a Cavarero, 2003.

${ }^{6}$ Sulla critica al contrattualismo, la letteratura è sterminata, ma è sempre attuale il classico di Pateman, Il contratto sessuale (1995). Per una riflessione sul comunitarismo, cfr. Molineux (1997). Spunti interessanti sui risvolti del comunitarismo nel multiculturalismo politico anche in Yuval Davis (1997).
} 
di organizzazione non oppressive, e quindi fonti di ispirazione per pensare un modello alternativo di cittadinanza e dell'agire politico. Le relazioni tra donne nate nei gruppi di autocoscienza degli anni '70, le comunità fondate sull'affidamento, la ricerca di genealogie femminili sono esempi di questa esplorazione. Alcune pensatrici hanno indirizzato la riflessione sul valore politico delle relazioni di amicizia - anche tra uomini e donne- come paradigma di una vita associata in cui sfera privata e sfera pubblica non sono scisse, e come esempio di una relazione basata "su uguaglianza, mutuo rispetto, cooperazione, sincerità, onestà, che sono poi le virtù repubblicane" (Forcina, 2003: 178). Nelle pagine di Delirio $y$ destino e in alcuni scritti autobiografici pubblicati nel volume Las palabras del regreso, evocando la Spagna degli anni 20 e 30, Zambrano offre suggestive annotazioni sulle relazioni di amicizia che nascevano all'interno dei gruppi giovanili impegnati nella creazione della "nueva política", in cui ella riconosceva la "vanguardia verdadera” di quel momento storico. E' rilevante il fatto che, a dimostrazione del carattere innovativo di quelle esperienze, Zambrano sottolinei proprio la sperimentazione di un inedito rapporto tra i sessi. Nella "alegría llena de gracia" dell'amicizia che si stabiliva tra ragazze e ragazzi, nel loro rifiuto degli schemi della seduzione, della civetteria, del dongiovannismo e della conquista, Zambrano coglie i germi di una politica autenticamente nuova, basata su un'idea dell'essere umano "libre de la ambición de poseer e irreductible a ser poseído” (Zambrano, 1998a: 224). ${ }^{7}$ Negli anni successivi, con lo sguardo disincantato prodotto dal naufragio non solo della Repubblica, ma anche di quelle comunità amicali, ${ }^{8}$ Zambrano torna a riflettere sull'amicizia tra i sessi mettendo in luce difficoltà e resistenze che derivano dalle sue implicazioni rivoluzionarie, sul piano culturale, morale e politico. Lo fa soprattutto in quel capolavoro che è "Eloisa o la existencia de la mujer". Attraverso la vicenda esemplare di Eloisa ed Abelardo, Zambrano indaga l'ideale senechiano di amicitia e la possibilità della sua trasposizione al rapporto tra i sessi, e argomenta che questo rappresenta una vera e propria audacia metafísica, poiché richiede il mutuo riconoscimento tra esseri diversi ma collocati su un piano di parità, e

\footnotetext{
${ }^{7}$ E' anche suggestivo approssimare l'affermazione di Zambrano secondo cui le donne e i giovani rappresentano "la vanguardia verdadera" per la loro "virginidad política" (Zambrano, 1998b: 510) con la convinzione che Carla Lonzi maturerà 40 anni dopo, nel pieno della rivolta del femminismo radicale: che si potesse stabilire una alleanza tra la donna e il giovane in chiave anti-patriarcale e che su questa alleanza fosse possibile profilare la politica nuova. Anche se lo sguardo smaliziato di Lonzi coglie perfettamente l'ambivalenza dei giovani maschi nel loro conflitto con il padre, alla cui sostituzione, nel ruolo di patriarchi, si candidano. Vedi il sempre attualissimo Lonzi, 1977).

${ }^{8}$ Del tramonto di quel cameratismo, e della resistenza da parte degli intellettuali della generazione del '27 a riconoscere, una volta in esilio, il ruolo e il carisma che Zambrano aveva esercitato sulla gioventù madrilena degli anni 20 e 30, parla Jesús Moreno Sanz (1996).
} 
dunque la sovversione del dualismo gerarchico della differenza tra i sessi (Zambrano, 1995:103).

Rilevante è l'annotazione sulla valenza politica che assume il rapporto tra uomini e donne se riscattato dalla coazione a ripetere insita nella forma matrimoniale istituzionale, in cui l' "heterogeneidad" dei soggetti sancisce l'incomunicabilità, e dunque non può che generare "la repetición". Se sperimentato nei termini dell'amicizia, l'amore può invece recuperare la forza creativa e trasformatrice, generatrice di mondi, che è propria di eros. La relazione tra i sessi trascende allora l'ambito del privato per sfociare nella inaugurazione "de un nuevo orden, de un nuevo reino".

\section{Il lavoro come cittadinanza/la cittadinanza nel lavoro}

La riflessione delle donne ha frequentemente portato l'attenzione sulla stretta connessione che vincola la cittadinanza (e in particolare la cittadinanza femminile) al lavoro. Il lavoro, ha affermato Lia Cigarini (2006: 33), è lo spazio pubblico per eccellenza, "la vera polis". Esso è infatti fonte di riconoscimento e di trasformazione, luogo di appartenenza e forma di azione nel mondo. Per le donne, l'ingresso nel mondo del lavoro extra-domestico è stato, almeno a partire dal secolo XIX, una modalità di presenza e di azione nello spazio pubblico che ha parzialmente colmato le lacune e il deficit della piena espressione della cittadinanza femminile. A questo proposito, Marisa Forcina (2006: 37) osserva acutamente che "scissa rispetto alla questione del lavoro, la questione della cittadinanza femminile è ancora quasi un ossimoro".

Nell'opera di María Zambrano possiamo trovare diversi spunti per pensare il valore politico del lavoro e il suo significato ambivalente ai fini del pieno dispiegamento della cittadinanza femminile. In primo luogo, c'è un'attenzione alla cosiddetta "sfera riproduttiva" e una messa in questione della separazione tra ambito pubblico e ambito privato che implica, come è noto, il mancato riconoscimento dell'attività di cura come lavoro e la dequalificazione della stessa, sia sul piano formale, sia su quello economico, sia su quello simbolico. Il riferimento ricorrente alle balie, alle nutrici, alle "serve" manifesta l'attenzione e l'alta considerazione in cui Zambrano teneva il mondo "de lo doméstico" che ella arriva a definire non solo "el cimiento", ma "el sujeto real de la historia": "aquel fondo de vida, aquella 'sustancia"” dalla quale i fatti storici trascendono; "lo que queda oculto bajo esta trascendencia” (Zambrano, 1982: 113). Questa rotonda affermazione suppone una valorizzazione della cura non solo sul piano materiale e strettamente economico, ma su quello politico e simbolico: implica cioè il riconoscimento del significato politico della cura come contributo al mondo e al bene comune. Nelle pagine che Zambrano dedica a queste figure, collocate nel

\footnotetext{
${ }^{9}$ M. Zambrano, "Sobre el mito de Tristán e Iseo", inédito. Fundación María Zambrano, VélezMálaga, M.15.
} 
piano più infimo della scala gerarchica delle professioni, appare evidente che per lei il lavoro domestico non è quello discreditato come mera "riproduzione" biologica (nella falsa convinzione che esista una tal cosa), quanto piuttosto attività complessa in cui si insegna, si cura, si trasmette, si rigenera e si inventa. Queste donne anonime creano, di fatto, la vita attraverso innumerevoli "trabajos sin nombre [...] que no alcanzan a ser un oficio, una profesión reconocida, una carta de ciudadanía" (Zambrano, 1982: 94).

Zambrano è anche attenta alle problematiche derivate dalla partecipazione femminile al lavoro extra-domestico, tanto sul piano dell'esistenza personale come su quello della vita pubblica. In uno degli articoli giovanili scritti per la rubrica "Mujeres" del periodico El liberal, s'interroga sulla invisibilità delle donne operaie nelle rappresentanze sindacali e nel discorso politico del partito socialista (alle cui posizioni era vicina), constatandone l'assenza "como clase y como sexo", e l'esclusiva apparizione in qualità di "ornato, presea o adorno" nelle manifestazioni (Zambrano, 1998b: 515). In un altro articolo denuncia il peso della doppia presenza, chiamando l'attenzione sul dramma delle madri costrette ad abbandonare i figli per accudire al lavoro nei campi o in fabbrica (Zambrano, 1998b: 511-512). Anche sul piano del simbolico e della rappresentazione, Zambrano è ben cosciente delle insidie presenti nel riferimento esclusivo dell'emancipazione e della libertà femminile allo svolgimento del lavoro extradomestico. Questo implica infatti la sopravvalutazione dei parametri che definiscono il lavoro maschile (salario, potere decisionale eccetera) e la sottovalutazione o il misconoscimento del valore e delle qualità specifiche del lavoro tradizionale femminile. Come ha ben sintetizzato Carole Pateman, evocando il "Dilemma Wollstonecraft", "L'accezione patriarcale della cittadinanza implica che le due domande siano incompatibili, poiché si danno solo due alternative: o le donne si trasformano in uomini, divenendo così cittadini in senso compiuto; oppure continuano a svolgere il loro lavoro di donne, che non ha alcun valore per la cittadinanza" (Pateman, 1989: 196, traduzione mia). In "Eloisa o la existencia de la mujer" Zambrano arriva a una sintesi altrettanto efficace quando, riferendosi al femminismo della propria epoca e alla sua enfasi nell'emancipazione, denuncia che questo condanna le donne alla scelta tra "una desdichada maternidad" e un "banal intento de independencia" (Zambrano, 1995: 92).

Per concludere, vorrei soffermarmi sul valore dellopera e della stessa vicenda personale di Zambrano come contributo alla riflessione sulle problematiche del lavoro intellettuale femminile. Zambrano è infatti un esempio paradigmatico di quelle che Virginia Woolf, in Le tre ghinee, chiama "le figlie degli uomini colti". E" una femme de lettres, che non solo fa della scrittura una professione e un mezzo di sussistenza, ma la concepisce come un modo di intervenire nel mondo e di trasformarlo. Sperimenta, di conseguenza, la contraddizione (e ne accetta la sfida) di assumere la posizione di soggetto del discorso emancipandosi da quella di 
oggetto, dovendo per di più operare con gli strumenti di un linguaggio forgiato da menti maschili. Lambivalenza di tale posizione appare chiaramente inquadrata negli scritti degli anni '40 (soprattutto Zambrano, 1940, a,b,c), dove Zambrano denuncia la difficoltà che trovano le donne intellettuali (ma lo stesso potremmo dire delle politiche) ad affermarsi a un tempo come professioniste e come donne vedendo quindi riconosciuta la propria individualità senza veder cancellata la propria femminilità-. Il suo "maestro", Ortega, offre una limpida prova della questione: nonostante contasse tra i suoi discepoli e tra le sue amicizie figure illustri di intellettuali donne - come la stessa María Zambrano, Rosa Chacel o Victoria Ocampo- che egli stimava e valorizzava, poteva scrivere, senza realizzare il paradosso: "La personalidad de la mujer es, más bien, un género que un individuo [...] El fondo personal de las almas femeninas es, poco más o menos, idéntico [...] La mujer es, para el pintor como para el amante, una promesa de individualidad que nunca se cumple" (Ortega, 2008: 154). La questione rimanda a quanto già detto sulla difficoltà che sperimentano le donne a essere riconosciute come "soggette", portatrici di proposte e visioni autonome, e non come espressione di un generico femminile. A questo proposito, Zambrano osserva acutamente che, quando esse rompono il recinto del mondo domestico per conquistare un posto nella cultura, sembrano esistere nella "ambiguedad de los seres sin sexo": appaiono creature fantasmagoriche, come le donne del Rinascimento italiano (1940a); o, come George Sand, si vedono costrette a vestire panni maschili, accettando, "con femenina docilidad", lequivalenza tra libertà e "mascolinismo" (1943: 311).

"Eloisa o la existencia de la mujer" è un saggio particolarmente ricco di spunti per approfondire la questione della ambigua posizione delle donne nella cultura, anche a motivo dei suoi chiari richiami autobiografici. Zambrano, infatti, sentiva fortemente l'identificazione con questa donna colta, innamorata della parola, sedotta dal pensiero. Definiva Eloisa una figura "pionera", come lo fu ella stessa; entrambe, infatti, aprirono con la forza perseverante della scrittura, un varco nel muro eretto a protezione della cittadella maschile del sapere per conquistarvi una collocazione, "una sede": un diritto di cittadinanza. E lo fecero "sin desmentirse", senza venir meno alla propria differenza, anche a costo di pagare con l'emarginazione o la reclusione: dalla propria dolorosa esperienza estrassero, come in una riuscita alchimia, una parola non gregaria, articolata in forme non convenzionali e in concetti non stereotipati, e per questo capace di imporsi alla nostra attenzione non in forza della legge del mercato del sapere, delle qualifiche e delle gerarchie, ma in virtù di quella legge non scritta, e ancora tutta da scrivere, che veicola la cittadinanza delle donne nel mondo.

\section{TESTI CITATI}

Ales Bello, Angela (1992), Fenomenologia dell'Essere Umano. Lineamenti di una filosofia al femminile, Roma, Città Nuova. 
Bimbi, Franca e Alisa Del Re (eds.) (1997), Genere e democrazia. la cittadinanza delle donne a cinquant'anni dal voto, Torino, Rosemberg e Sellier.

Birulés, Fina (2007), Hannah Arendt. Una herencia sin testamento, Barcellona, Herder.

-(2014), Entreactes. Entorn a la política, el feminisme i el pensament, Canet de Rosselló, Edicions Trabucaire.

Boccia, Maria Luisa (2002), La differenza politica. Donne e cittadinanza, Milano, Il Saggiatore.

Bonacchi, Gabriella e Angela Groppi (eds.) (1993), Il dilemma della cittadinanza. Diritti e doveri delle donne, Roma-Bari, Laterza.

Castells, Carme (ed.) (1996), Perspectivas feministas en teoría politica, Barcellona, Paidós.

Cavarero, Adriana (2003), A più voci. Filosofia dell'espressione vocale, Milano, Feltrinelli.

Cigarini, Lia (2006), "Un'altra narrazione del lavoro", Critica Marxista, 6: 33-36.

Collin, Françoise (1992a), "Praxis de la différence. Note sur le tragique du sujet", Les Cahiers du Grif (Provenances de la pensée. Femmes/Philosophie): 125-141.

-(1992b), "Agir et donné”, Hannah Arendt et la modernité, A.M. Roviello e M. Weyembergh (eds.), Paris, Vrin.

-(1997), "La disputa della differenza. La differenza tra i sessi e il problema delle donne in filosofia", Storia delle donne. Il novecento, George Duby e Michelle Perrot (eds.), Bari, Laterza: 306-343.

Del Re, Alisa e Jaqueline Heinen (eds.) (1996), Quale cittadinanza per le donne?, Milano, Franco Angeli.

Dietz, Mary G. (1985), "Citizenship with a Feminist Face: The Problem of Maternal Thinking”, Political Theory, 13.1: 19-37.

Forcina, Marisa (2000), Soggette. Corpo, politica, filosofia: percorsi nella differenza, Milano, Franco Angeli.

-(2003), Una cittadinanza di altro genere, Milano, Franco Angeli.

-(2006), "Donne: lavoro e cittadinanza", Critica Marxista, 6: 37-44.

-(2009), Rappresentazioni politiche della differenza, Milano, Franco Angeli.

Friedman, Marilyn (1993), "Feminism and Modern Friendship: Dislocating the Comunity”, Friendship: A Philosophical Reader, Neera Badhwar Kapur (ed.), Ithaca, Cornell University Press.

Libreria delle donne di Milano (1987), Non credere di avere dei diritti, Milano, La Tartaruga.

Lonzi, Carla (1977), Sputiamo su Hegel. La donne clitoridea e la donna vaginale, Milano, Scritti di Rivolta Femminile. 
Molineux, Maxine (1997), "Debates sobre comunitarismo, moralidad y políticas de identidad”, La ciudadanía a debate, Eugenia Hola e Ana María Portugal (eds.), Santiago de Chile, Isis Internacional.

Moreno Sanz, Jesús (1996), "La política desde su envés histórico-vital. Historia trágica de la esperanza y sus utopías”, Horizonte del liberalismo, Madrid, Morata: 9-12.

Ortega y Gaset, José (2008), Obras completas: 1926-1932, volume 8, Madrid, Taurus.

Pateman, Carole (1989), "The Pathriarcal Welfare State", The disorders of women, Cambridge, Polity Press.

Scott, Joan W. (1996), Only Paradoxes to Offer. French Feminists and the Right of Man, Cambridge, Harvard University Press.

Stein, Edith, (1987), La donna. Il suo compito secondo la natura e la grazia, Roma, Città Nuova.

Young, Iris Marion (1994), Feminism and Political Theory, Chicago, The University of Chicago Press.

Yuval Davis, Nira (1997), Gender \& Nation, London, Sage.

Zambrano, María (1940a), "La mujer en la cultura medieval”, Ultra, 45: 275-278.

-(1940b), "La mujer en el Renacimiento", Ultra, 46: 367-369.

-(1940c), "La mujer en el Romanticismo", Ultra, 46: 368-369.

-(1943), "La mujer en la historia", Universidad de La Habana, 49: 310-317.

-(1982), La España de Galdós, Barcellona, La Gaya Ciencia.

-(1995), "Eloísa o la existencia de la mujer", María Zambrano. Nacer por sí misma. Ensayos sobre Antígona, Eloisa, Diótima, Elena Laurenzi (ed.), Madrid, horas y HORAS: 90-113.

-(1996), Horizontes del liberalismo, Madrid, Ediciones Morata.

-(1998a), Delirio y destino, Madrid, Editorial Centro de Estudios Ramón Areces.

-(1998b), "Mujeres" (rubrica de El Liberal Madrid, 2 de agosto-8 de noviembre), en Juan Carlos Marset, "Los artículos de María Zambrano en "Aire Libre", Actas del II Congreso Internacional sobre la Vida y la Obra de María Zambrano, Vélez-Málaga, Fundación María Zambrano: 508-518. [1928]

-(1999), “A propósito de la 'grandeza y servidumbre de la mujer”, Aurora. Papeles del "Seminario María Zambrano", 1: 143-149.

-(2006), Persona y democracia, Madrid, Siruela. 